\title{
Irradiation uniformity at the Laser MegaJoule facility in the context of the shock ignition scheme
}

\author{
Mauro Temporal ${ }^{1}$, Benoit Canaud ${ }^{2}$, Warren J. Garbett ${ }^{3}$, Rafael Ramis ${ }^{4}$, and Stefan Weber ${ }^{5}$ \\ ${ }^{1}$ Centre de Mathématiques et de Leurs Applications, ENS Cachan and CNRS, 61 Av. du President Wilson, Cachan Cedex, France \\ ${ }^{2}$ CEA, DIF, Arpajon Cedex, France \\ ${ }^{3}$ AWE plc, Aldermaston, Reading, Berkshire, United Kingdom \\ ${ }^{4}$ ETSI Aeronáuticos, Universidad Politécnica de Madrid, Madrid, Spain \\ ${ }^{5}$ ELI-Beamlines, Institute of Physics, Academy of Sciences of the Czech Republic, Prague, Czech Republic \\ (Received 27 February 2014; revised 24 March 2014; accepted 31 March 2014)
}

\begin{abstract}
The use of the Laser MegaJoule facility within the shock ignition scheme has been considered. In the first part of the study, one-dimensional hydrodynamic calculations were performed for an inertial confinement fusion capsule in the context of the shock ignition scheme providing the energy gain and an estimation of the increase of the peak power due to the reduction of the photon penetration expected during the high-intensity spike pulse. In the second part, we considered a Laser MegaJoule configuration consisting of 176 laser beams that have been grouped providing two different irradiation schemes. In this configuration the maximum available energy and power are $1.3 \mathrm{MJ}$ and $440 \mathrm{TW}$. Optimization of the laser-capsule parameters that minimize the irradiation non-uniformity during the first few ns of the foot pulse has been performed. The calculations take into account the specific elliptical laser intensity profile provided at the Laser MegaJoule and the expected beam uncertainties. A significant improvement of the illumination uniformity provided by the polar direct drive technique has been demonstrated. Three-dimensional hydrodynamic calculations have been performed in order to analyse the magnitude of the azimuthal component of the irradiation that is neglected in twodimensional hydrodynamic simulations.
\end{abstract}

Keywords: inertial confinement fusion; shock ignition; laser system

\section{Introduction}

One of the main goals of inertial confinement fusion $(\mathrm{ICF})^{[1-3]}$ concerns the ignition of the thermonuclear fusion reactions in a mixture of deuterium-tritium (DT) nuclear fuel. After the ignition phase, it is expected that propagation of a thermonuclear burn wave - dominated by the fusion reaction with larger cross section $D+T=>$ $\alpha+n+17.6 \mathrm{MeV}-$ throughout the compressed fuel should generate a large energy gain $G=E_{\text {fus }} / E_{\text {in }}$ (ratio between thermonuclear fusion and the invested energy). To this aim two schemes have been proposed, namely: direct drive and indirect drive. In both cases a spherical capsule containing the DT nuclear fuel is considered. In the direct drive ${ }^{[4]}$ scheme the spherical capsule is irradiated by a large number of laser beams, whilst in the indirect drive ${ }^{[1]}$ scheme the laser energy is first converted into an x-ray field (confined into a high- $Z$ casing; see hohlraum) that irradiates the

Correspondence to: Mauro Temporal, Centre de Mathématiques et de Leurs Applications, ENS Cachan and CNRS, 61 Av. du President Wilson, Cachan Cedex, France. Email: mauro.temporal@hotmail.com capsule. The energy deposited in the external capsule shell provides a series of strong shock waves that induces the capsule implosion. In the classical central ignition scheme ${ }^{[5]}$ the DT fuel is accelerated to high implosion velocities (hundreds of $\mathrm{km} \mathrm{s}^{-1}$ ) before stagnating to produce a highdensity (hundreds of $\mathrm{g} \mathrm{cm}^{-3}$ ) shell that confines a fraction of the DT fuel (hundreds of $\mu \mathrm{g}$ ). The ignition conditions require that the central mass, called the hot-spot, is heated to high temperature $(10 \mathrm{keV})$ and confined into a volume with an areal density comparable with the $\alpha$-particle range $\left(\approx 0.3 \mathrm{~g} \mathrm{~cm}^{-2}\right)$.

A crucial issue concerns the uniformity of the capsule irradiation. A successful capsule implosion requires a very uniform irradiation and capsule target; otherwise, the imploding shell suffers the growth of dangerous hydrodynamic instabilities (Richtmyer-Meshkov ${ }^{[6,7]}$ and Rayleigh-Taylor $(\mathrm{RT})^{[8,9]}$ ) and shell deformations that could even destroy the hot-spot. A way to reduce the growth of RT instability consists of compressing the capsule fuel at low implosion velocity $V$. This causes a detriment of the energy gain $G$ which scales $^{[10]}$ as $\varphi /\left(V^{5 / 4} I^{1 / 4}\right)$, where $\varphi=\rho R /(\rho R+7)$ 


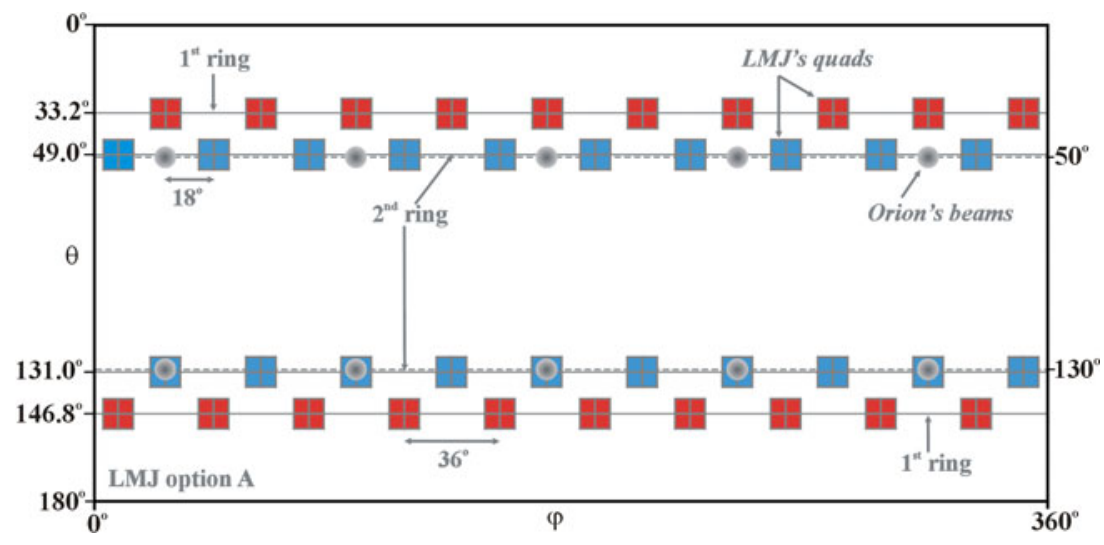

Figure 1. Angular coordinate of the 40 quads (blue and red boxes) distributed to the first and second ring of the LMJ facility. The gray circles represent the polar coordinates of the 10 long-pulse beams of the Orion facility.

is the fractional burn-up ${ }^{[1,3]}$ with $\rho R$ the fuel areal density, and $I$ the incident laser intensity.

Alternative schemes are currently under study, such as fast ignition induced by laser accelerated electrons ${ }^{[11,12]}$, protons ${ }^{[13-15]}$, or heavier ions ${ }^{[16,17]}$. More recently, the shock ignition (SI) scheme $e^{[18,19]}$ has been proposed as an alternative to the classical central ignition in the context of the inertial confinement fusion scenario. In this case, the capsule is directly irradiated by the laser beams providing the compression of the DT fuel. The implosion velocity of the compressed shell is set under the ignition threshold $\left(V<2-3 \times 10^{7} \mathrm{~cm} \mathrm{~s}^{-1}\right)$ and does not allow for the generation of an efficient hot-spot. In the SI scheme, a second high-power (hundreds of TW) laser pulse irradiates the capsule and drives a strong shock wave that reaches the compressed shell providing the fuel ignition. The SI pulse must be carefully tuned in time to synchronize the strong shock wave with the compression shock rebounded from the centre after stagnation. This new scheme promises higher gain ${ }^{[18-21]}$ in comparison to central ignition, and the separation between the compression and the ignition phase allows for less stringent conditions in terms of irradiation uniformity ${ }^{[22,23]}$. Moreover, this two-step irradiation would benefit also from the zooming technique ${ }^{[24,25]}$ in order to increase the laser-capsule coupling efficiency. Nevertheless, caution is necessary due to the uncertainties related to laserplasma instabilities such as stimulated Raman scattering $(\mathrm{SRS})^{[26]}$, stimulated Brillouin scattering (SBS) ${ }^{[27]}$, and the two-plasmon decay (TPD) ${ }^{[28]}$ expected at the high laser intensities $I \lambda^{2}>10^{15} \mathrm{~W} \mathrm{~cm}^{-2} \mu \mathrm{m}^{2[29]}$ provided during the shock ignition pulse. These dangerous instabilities act to reduce the energy deposition efficiency and generate highenergetic $(\approx 10-40 \mathrm{keV})$ electrons ${ }^{[30-32]}$. The uncertainties concerning the laser-plasma interaction correlated to the shock ignition scheme have also motivated great interest in experimental activities ${ }^{[33-39]}$. Moreover, large laser facilities such as the National Ignition Facility (NIF) ${ }^{[40,41]}$ in the USA and the Laser MegaJoule (LMJ) facility ${ }^{[2,43]}$ in France as well as the smaller Orion facility ${ }^{[44]}$ in the UK - all of them devoted to the indirect drive scheme - could be used to test relevant aspects inherent to the shock ignition scheme.

Due to its indirect drive design, the LMJ facility does not provide a favourable laser beam configuration for direct drive irradiation. Nevertheless, this large facility is very attractive for direct drive studies because of its large available energy. In this context, this paper aims to chart a path starting from the current characteristics of the LMJ facility and exploring the potential of the shock ignition scheme. After summarizing the main characteristics of the LMJ facility in Section 2, the paper analyses two different aspects: the requirement in the maximum power on the shock ignition scheme together with possible consequences due by laser-plasma instabilities in Section 3, and the study of the uniformity of the irradiation during the foot pulse of the imprint phase in Section 4, while in Section 5 some three-dimensional (3D) aspects of the hydrodynamics of the implosion are discussed. Then, conclusions are drawn in Section 6.

\section{The Laser MegaJoule configuration}

The configuration of the Laser MegaJoule facility considered in this paper consists of 176 high-power laser beams. These beams are grouped in 44 identical quads, each one composed by four beams. 40 quads are distributed into the spherical experimental chamber in four axial symmetric rings, and the two hemispheres are rotated by $18^{\circ}$. The two rings closer to the polar axis have an angle of $33.2^{\circ}$ and $146.8^{\circ}$ with each having 10 quads; another 20 quads are located in the rings at $49^{\circ}$ and $131^{\circ}$, as shown in Figure 1. The last four quads, not shown in the figure, will be located in two additional rings at $59.5^{\circ}$ and $120.5^{\circ}$. The LMJ architecture is designed to provide a maximum laser energy (power) of $7.5 \mathrm{~kJ}(2.5 \mathrm{TW})$ for each beam. Consequently, this corresponds to a total energy (power) of $30 \mathrm{~kJ}(10 \mathrm{TW})$ per quad, and each pair of rings will provide a maximum energy (power) of $600 \mathrm{~kJ}$ 


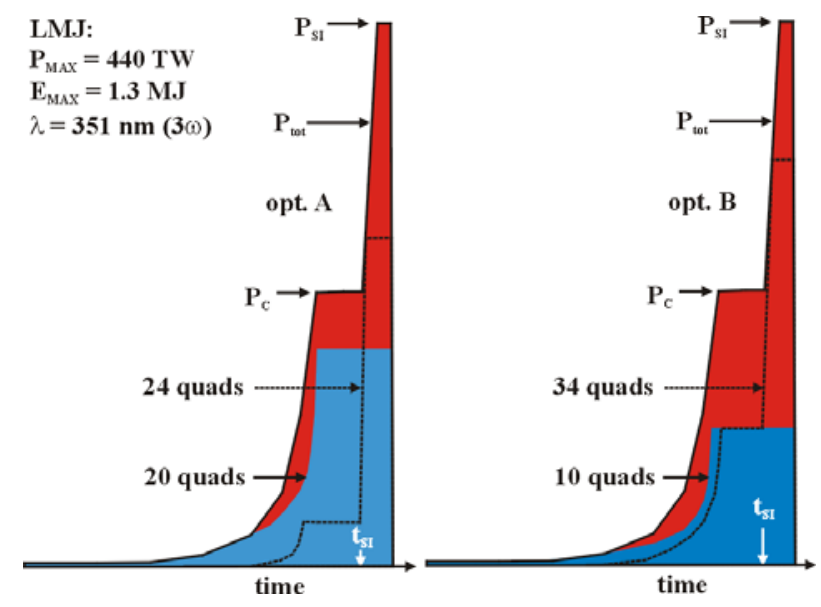

Figure 2. Sketch of the temporal power profile partition for the two LMJ options, A (left) and B (right), in the shock ignition scheme.

(200 TW). This makes the LMJ a large laser facility, able to drive a total energy of about $1.3 \mathrm{MJ}$ with a maximum power of $440 \mathrm{TW}$ delivered by laser beams with a wavelength of $\lambda=351 \mathrm{~nm}(3 \omega)^{[45]}$. The facility design energy is appropriate for indirect drive central ignition, but both direct drive and shock ignition schemes are expected to lower the energy threshold for ignition. Thus the total available energy at the LMJ facility largely exceeds the needs in the direct drive approach.

The polar coordinates of the quads have been optimized for the indirect drive scheme. Nevertheless, this laser beam distribution could be helpful also in the direct drive shock ignition scheme. Indeed, as already mentioned, this scheme involves two laser pulses: one for the capsule compression and a second one for the fuel ignition. Thus, there are several options in order to use the LMJ facility as a direct drive facility in the context of the SI scheme. Hereafter, we will consider two options: option A, where 20 quads of the second ring are devoted to the compression of the capsule and with the other 24 quads driving the high-power shock ignition pulse; and option B, with only 10 quads of the second ring devoted to the foot pulse while together with the remaining 34 quads contributing to the drive and the igniting pulse. The main difference between the two options concerns the role of the different quads in the partition of laser power during the low-power foot pulse, the main drive of the compression phase, and the shock ignition phase.

Of course, the choice of the irradiation configuration also has consequences on the irradiation uniformity. Details of these configurations are given in the temporal power pulse sketched in Figure 2. In option A, the whole $600 \mathrm{~kJ}$ (200 TW) of the laser beams of the second ring are available for the compression phase and quads of the first ring are almost entirely associated to the shock ignition pulse. In option B, only $300 \mathrm{~kJ}$ (100 TW) - half the laser beams of the second ring - are devoted to the foot pulse and part of the compression phase, while part of the drive and the shock ignition pulse operate with the 34 quads located in the three rings. In both cases the maximum available power for the shock ignition pulse will be the totality of the $440 \mathrm{TW}$.

The division of tasks among the different quads also allows implementing in a natural way both the polar direct drive (PDD) ${ }^{[46,47]}$ and the zooming technique. The purpose of the PDD is to adapt a non-optimal configuration of laser beams in order to optimize the uniformity of the capsule irradiation. To this purpose, in the PDD technique the laser beam directions are modified in order to optimize the direct drive capsule irradiation ${ }^{[48-51]}$. The use - as a direct drive of the quads in the second rings of the LMJ facility leads to an over-irradiation of the capsule polar regions, whilst the equatorial area is under-irradiated. Thus, applying the PDD by displacing the quad toward the equator improves the capsule illumination uniformity considerably.

It is worth noting that the Orion facility in the UK is composed of 12 beams: two laser beams provide $500 \mathrm{~J}$ each at $1 \omega(1054 \mathrm{~nm})$ in a short pulse of $0.5 \mathrm{ps}$ and the other ten provide a total energy of $5 \mathrm{~kJ}(3 \omega, \lambda=351 \mathrm{~nm})$ in $1-5 \mathrm{~ns}$ long pulses. The angular positions of these ten beams are indicated by gray circles in Figure 1 . These $5+5$ beams are located in two rings at $50^{\circ}$ and $130^{\circ}$ with respect to the polar angle. This beam distribution is very similar to the one provided by the second ring of the LMJ facility. This makes the Orion facility the natural choice to test relevant aspects inherent to the LMJ facility such as the laser absorption and the improvement of the irradiation uniformity promised by the PDD technique. Indeed, despite the relatively small dimensions, $5 \mathrm{~kJ}$ in $5 \mathrm{~ns}$ for $1 \mathrm{TW}$, this facility is perfectly matched to the requirements of experiments dedicated to the study of the imprint phase, where the first shock wave is driven by a low-power $(\approx \mathrm{TW})$ foot pulse.

\section{Shock ignition calculations}

A relatively large direct drive capsule characterized by an initial aspect ratio $A=3$ has been considered. This capsule is part of a family of capsules that have been recently studied $^{[52]}$. This spherical capsule has an external radius of $815 \mu \mathrm{m}$ and contains a DT fuel mass of $300 \mu \mathrm{g}$. The cryogenic nuclear fuel $\left(\rho_{\mathrm{DT}}=0.25 \mathrm{~g} \mathrm{~cm}^{-3}\right)$ is surrounded by a thin $(24 \mu \mathrm{m})$ shell of plastic $\left(\rho_{\mathrm{CH}}=1.07 \mathrm{~g} \mathrm{~cm}^{-3}\right)$ devoted to the laser energy absorption. Detailed parametric studies have been performed showing that the self-ignition threshold in the implosion velocity is about $3 \times 10^{7} \mathrm{~cm} \mathrm{~s}^{-1[53]}$ and that the maximum energy gain is $G=44$ with an incident energy of about $500 \mathrm{~kJ}$ and laser peak power of $230 \mathrm{TW}^{[54]}$. In the considered LMJ design, the available energy (1.3 MJ) is above that needed by the capsule, which means we retain some energy margin against constraints mainly related to the required irradiation uniformity.

Here, the capsule has been used in the context of the shock ignition scheme, and a series of 1D numerical calculations has been performed with the hydro-radiative MULTI 
code ${ }^{[55,56]}$. In this version, the MULTI code takes into account the tabulated equation of state, heat conduction (Spitzer-Harm flux limited 8\%) and a 3D ray-tracing package that manage the laser energy absorption via an inverse bremsstrahlung mechanism. A laser beam characterized by a Gaussian intensity profile with a full-width at halfmaximum of $1356 \mu \mathrm{m}$ - the intensity is reduced to $1 / e$ at the initial capsule radius - has been considered. The laser pulse is composed of a low-power foot pulse $(\approx 7 \mathrm{~ns}$ at $\approx \mathrm{TW}$ ) followed by the main pulse with a maximum power $P_{\mathrm{C}}=180 \mathrm{TW}$ (see the shadow area in Figure 3) which drives the capsule compression. This laser pulse does not provide self-ignition but only serves to compress the DT fuel. Indeed, ignition is achieved by the action of an additional high-power shock ignition spike. In this case, the igniting SI pulse starts at time $t_{\mathrm{SI}}$ and the power grows linearly, reaching the maximum power $P_{\mathrm{SI}}$ in $100 \mathrm{ps}$; the maximum power holds for 300 ps and then goes down to zero in another 100 ps.

In Figure 3 are shown details of a numerical calculation performed with a compression pulse which is maintained until time $t_{\mathrm{SI}}=12.6 \mathrm{~ns}$, where the shock ignition pulse began. The maximum incident laser power during the compression phase is $P_{\mathrm{C}}=180 \mathrm{TW}$, which then grows to $P_{\mathrm{SI}}=350 \mathrm{TW}$ during the shock ignition pulse. In this case, the calculation provides a fusion energy of $E_{\mathrm{FUS}}=24.3 \mathrm{MJ}$ while the total incident laser energy is $E_{\mathrm{INC}}=560 \mathrm{~kJ}$, of which $360 \mathrm{~kJ}$ are invested in the compression phase $\left(t<t_{\mathrm{SI}}\right)$ and $200 \mathrm{~kJ}$ in the shock ignition pulse $\left(t>t_{\mathrm{SI}}\right)$. The energy gain is $G=E_{\mathrm{FUS}} / E_{\mathrm{INC}}=43$. The incident and absorbed laser powers are shown by the two shadowed areas (that correspond to the linear scale) in Figure 3. As can be seen, the absorbed power is almost the half of the incident laser power, and the total energy absorption fraction is $\eta=59 \%$. It is worth noticing that this capsule provides similar performance when is used in the central ignition scheme. Nevertheless, the shock ignition scheme benefits from a larger tolerance with respect to the fuel compression uniformity, which is the drawback at the LMJ facility.

The radial position $r_{\mathrm{c}}$ where the density is equal to the critical value $\rho_{\mathrm{c}}\left[\mathrm{g} \mathrm{cm}^{-3}\right]=1.865 \times 10^{-3}(A / Z) / \lambda^{2}[\mu \mathrm{m}]$ has also been calculated, and it is shown by the dashed red curve in Figure 3. The surface associated with the radius $r_{\mathrm{c}}$ has been used to estimate the maximum incident laser intensity $I_{\mathrm{INC}}=P_{\mathrm{INC}} /\left(4 \pi r_{\mathrm{c}}^{2}\right)$, shown by the blue curve. As usual in the shock ignition scheme, a very high laser intensity $I_{\mathrm{INC}}$ is needed during the shock ignition pulse, but there is some concern that the laser-plasma interaction at high intensities $\left(I \lambda^{2}>10^{15} \mathrm{~W} \mathrm{~cm}^{-2} \mu \mathrm{m}^{2}\right)$ is dominated by laser-plasma instabilities that considerably modify the absorption mechanism ${ }^{[57]}$ and negatively impact coupling of the ignition pulse energy to the capsule. In our case, the maximum intensity is larger than $10^{16} \mathrm{~W} \mathrm{~cm}^{-2}$, although the true value may not be quite this high, since not all the incident power $P_{\mathrm{INC}}$ reaches the critical density surface, also

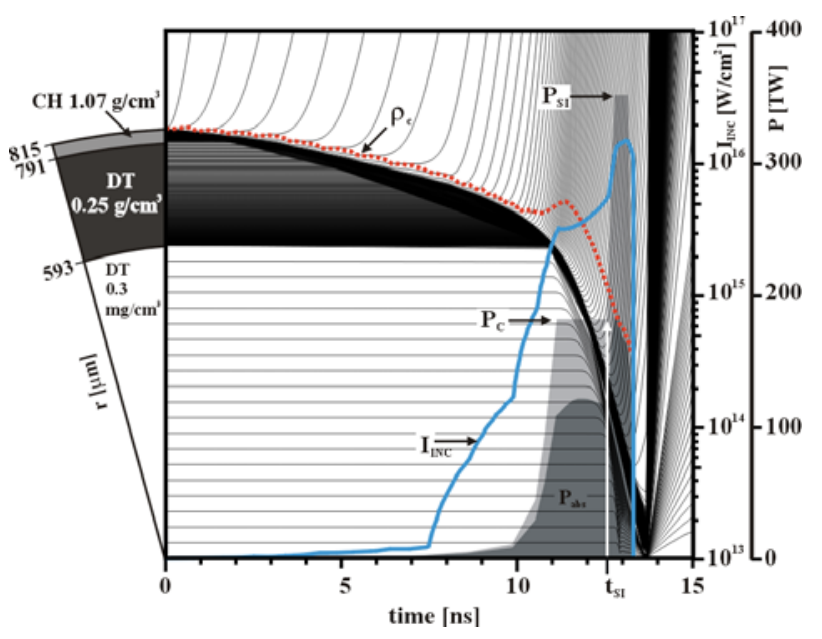

Figure 3. Capsule dimensions and temporal evolution of the Lagrangean radii. The temporal profile of the incident and absorbed power are shown by the two shadowed areas. The position of the critical density $\left(\rho_{\mathrm{c}}\right)$ and evolution of the maximum incident laser intensity $\left(I_{\mathrm{INC}}\right)$ are also shown as a function of time.

due to beam refraction. Nevertheless, these intensities are still in excess of the thresholds for laser plasma instabilities. It is likely that a large part $(\approx 50 \%)$ of the photon energy is converted into energetic electrons $(\approx 30 \mathrm{keV})$ and the laser light does not penetrate until the classical critical density, $\rho_{\mathrm{c}}$, but reaches only $\rho_{\mathrm{c}} / 4^{[58-61]}$ where the laser light is absorbed by collective effects. The physics involving these high laser intensities and electronic transport are not included in our hydrodynamic code. Nevertheless, we tried to mimic the reduced critical density assuming a laser wavelength $\left(\lambda_{\mathrm{SI}}\right)$ twice the nominal value $\left(\lambda_{\mathrm{SI}}=2 \lambda=702 \mathrm{~nm}\right)$ during the shock ignition pulse, i.e., for $t>t_{\text {SI }}$. Of course, this is just an attempt to evaluate the effect of the reduction by a factor of four $\left(\rho_{\mathrm{c}} \alpha \lambda^{-2}\right)$ in the maximum density reached by the photons. Detailed hydrodynamic calculations that also include the high-energetic electron transport will be needed to give a more complete treatment of the problem.

Two parametric studies have been performed, varying the starting time, $t_{\mathrm{SI}}$, of the shock ignition pulse and the maximum incident power, $P_{\text {SI }}$. In a first case, we used the usual laser wavelength $\lambda=351 \mathrm{~nm}$ during the whole calculations and for each couple of parameters, $t_{\mathrm{SI}}$ and $P_{\mathrm{SI}}$, the final energy gain $G$ has been calculated. In a second set of calculations the laser wavelength has been doubled during the shock ignition pulse - i.e., when $t<t_{\mathrm{SI}}-$ providing the gain $G^{*}\left(t_{\mathrm{SI}}, P_{\mathrm{SI}}\right)$. The colour maps in Figure 4 shown the gain $G$ and $G^{*}$ as a function of the two parameters $t_{\mathrm{SI}}$ and $P_{\mathrm{SI}}$. In the same figures the white contour curves show the total absorbed energy fraction, $\eta$. The case of the gain $G-$ Figure 4(a), evaluated using always the same wavelength $\lambda$ - shows two regions with high gain. In the first maximum, at smaller parameter $t_{\mathrm{SI}}$ and characterized by a lower gain, the high-power laser pulse arrives too early 


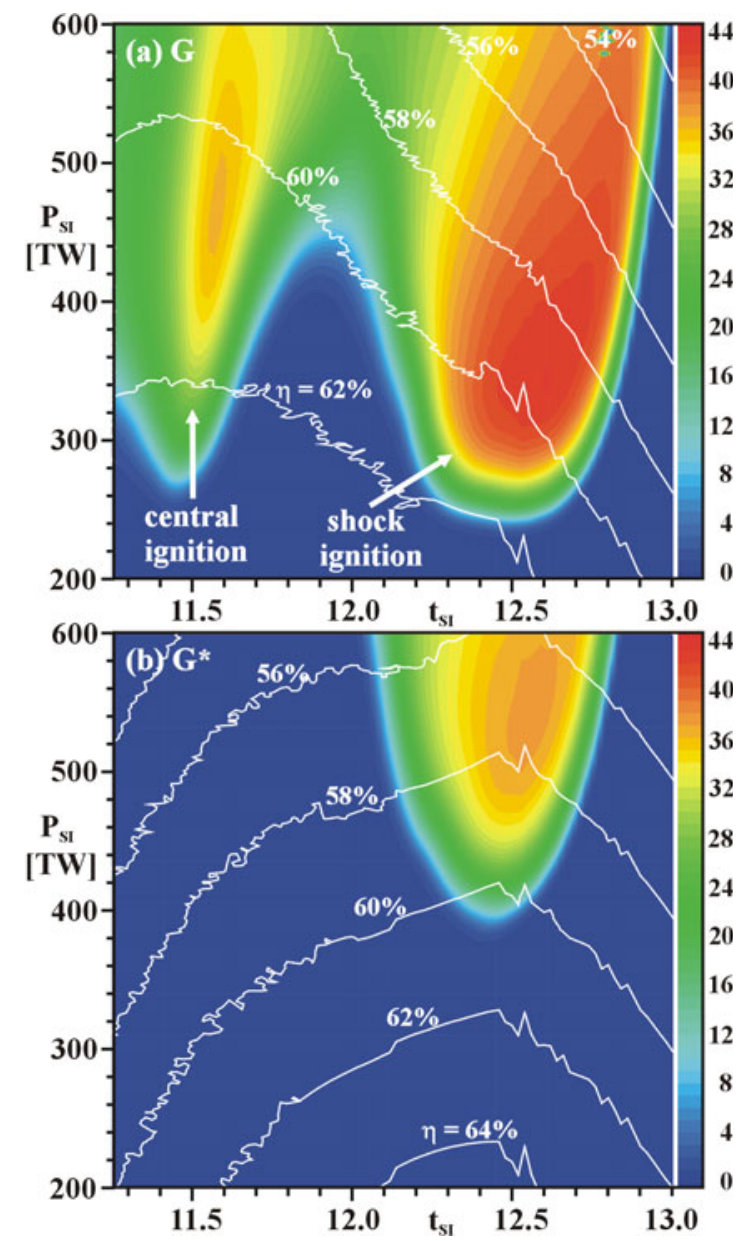

Figure 4. Gain as a function of the starting time $t_{\mathrm{SI}}$ and of the maximum power $P_{\mathrm{SI}}$ of the shock ignition pulse. (a) Gain $G$, calculated with $\lambda_{\mathrm{SI}}=\lambda$; (b) Gain $G^{*}$, calculated assuming $\lambda_{\mathrm{SI}}=2 \lambda$. The white curves represent isovalues of the absorption, $\eta[\%]$.

and generates a Kidder-like exponential laser pulse ${ }^{[62]}$ that induces the classical central ignition. In contrast, the shock ignition mechanism is responsible for the second stronger signal at larger times, $t_{\mathrm{SI}}$. It is found that for this specific laser-capsule configuration the threshold in the power $P_{\mathrm{SI}}$ is about $250 \mathrm{TW}$. The ignition region is reduced in the case of the gain $G^{*}$ when the laser wavelength has been artificially doubled during the shock ignition pulse. In this case, the threshold moves to higher powers at around $400 \mathrm{TW}$. In both cases, $G$ and $G^{*}$, the energy absorption is around $60 \%$, and the modification in the threshold comes from the fact that the laser energy is deposited far from the compressed fuel. We are aware that our calculations do not deal with the correct laser-plasma interaction mechanisms; thus these results are not conclusive and just indicate a trend. It is also worth noting that the energetic electrons, neglected in our calculations, can transport some energy ${ }^{[63,64]}$ between the deposition region $\left(\rho<\rho_{\mathrm{C}} / 4\right)$ and the ablation front that should favourably reduce the incident power threshold. In fact the high-energy electrons may not be as detrimental as in the central ignition scheme, and it is possible they may even contribute positively towards driving the ignition shock ${ }^{[65]}$.

\section{Illumination non-uniformity}

The shock ignition scheme is less demanding than the central ignition one with respect to the uniformity of the irradiation $^{[66,67]}$. However, the spike power needed to ignite the target is sensitive to the uniformity of the fuel assembly ${ }^{[59]}$, and it is necessary to control the irradiation uniformity during the whole duration of the laser pulse. This is in general also difficult, because the plasma corona evolves during this time, and laser parameters optimized at the beginning of the irradiation could be no longer appropriate later during the implosion ${ }^{[68]}$. Nevertheless, special care must be paid to minimize the initial irradiation non-uniformity that generates the first shock wave of the implosion and dominates the so-called imprint phase.

In this section, we analyse some of the behaviour of the irradiation by using the illumination model ${ }^{[69,70]}$. In the model, the capsule is assumed stationary - expansion of the plasma corona is neglected - and is characterized only by the external radius $r_{0}$. For a given number of incident laser beams characterized by an arbitrary laser intensity profiles, the model calculates the intensity of the illumination $I(\theta, \phi)$ over the spherical surface. It is thus assumed that laser parameters that optimize the illumination uniformity also minimize the non-uniformity transmitted to the first shock wave ${ }^{[71]}$. Generally, the quality of the illumination is measured by the root-mean-square deviation, $\sigma_{0}$, associated to the function $I(\theta, \phi)$; this is given by

$$
\sigma_{0}=\left\{\frac{1}{4 \pi} \int_{0}^{2 \pi} \int_{0}^{\pi}[I(\theta, \phi)-\langle I\rangle]^{2} \sin (\theta) d \theta d \phi\right\}^{1 / 2} /\langle I\rangle,
$$

where $\langle I\rangle$ is the average intensity over the surface of the spherical target. The intrinsic non-uniformity $\sigma_{0}$ is a characteristic of a given laser-capsule configuration and assumes perfectly ideal laser beams not affected by any imperfections.

In reality, laser beams suffer from unavoidable errors such as beam-to-beam power imbalance $\sigma_{\mathrm{PI}}$, laser pointing error $\sigma_{\mathrm{PE}}$, and error in the target positioning $\sigma_{\mathrm{TP}}$. These errors are statistical quantities that in the case of the LMJ facility are estimated by the standard deviations: $\sigma_{\mathrm{PI}}=10 \%$ (beam-to-beam), $\sigma_{\mathrm{PE}}=50 \mu \mathrm{m}$, and $\sigma_{\mathrm{TP}}=20 \mu \mathrm{m}$. In the LMJ facility, the laser beams are grouped in quads; thus the power imbalance benefits from a statistical factor which reduces it to $\sigma_{\mathrm{PI}}=5 \%$ (quad-to-quad). The illumination non-uniformity, evaluated taking into account these beam uncertainties, is usually measured as an average value $(\underline{\sigma})$ estimated over a large number of calculations ${ }^{[72-77]}$. In these calculations, each of the three parameters (laser power, laser 
pointing, and target position) varies randomly and follows a Gaussian distribution centred to their nominal values and characterized by the corresponding standard deviation $\sigma_{\mathrm{PI}}$, $\sigma_{\mathrm{PE}}$, or $\sigma_{\mathrm{TP}}$.

As has been already said, the LMJ facility is devoted to the indirect drive scheme. This means that the laser beam directions as well as their intensity profile fit with the hohlraum requirements. As a consequence, the geometrical shape of the laser intensity profile is elliptical. Thus, the intensity profile has been parameterized by the super-Gaussian function: $I(x, y)=I_{0} \exp -\left[\left(x / \Delta_{\mathrm{a}}\right)^{2}+\left(y / \Delta_{\mathrm{b}}\right)^{2}\right]^{m / 2}$, characterized by the parameters $\Delta_{\mathrm{a}}$ and $\Delta_{\mathrm{b}}$ (half width at $1 / e$ ) and by the exponent $m$. In our calculations we considered an elliptical laser intensity profile characterized with $\Delta_{\mathrm{a}}=2 \Delta_{\mathrm{b}}, \Delta_{\mathrm{b}}=$ $320 \mu \mathrm{m}$, and an exponent $m=4$. Because of specific needs inherent to the indirect drive scheme in the LMJ facility (the same applies also for the Orion facility), the minor axis $\left(\Delta_{\mathrm{b}}\right)$ of the elliptical intensity profile is located in the meridian defined by the polar and beam axes.

In the first set of calculations we considered the nonuniformity provided by the 20 quads located in the second ring of the LMJ facility (option A in Section 2). The results are shown in the Figure 5 as a function of the target radius $r_{0}$. The cloud of dots in the figure represents the results obtained with the elliptical profile for a large number of calculations assuming a random Gaussian distribution for the power imbalance, pointing error, and target positioning. The continuous red curve is the average non-uniformity $\underline{\sigma}$, while the blue curve shows the intrinsic non-uniformity $\sigma_{0}$ evaluated neglecting any beam-capsule uncertainties. As can be seen, for small capsule radii, some configurations with capsule centre, laser powers, and pointing randomly assigned can provide better results with respect to the intrinsic values. For the given elliptical LMJ laser intensity profile, an optimum capsule radius of $r_{0}=320 \mu \mathrm{m}$ is found for which the average non-uniformity assumes the minimum value $\underline{\sigma}=6.2 \%$, while the minimum intrinsic nonuniformity, evaluated neglecting any beam uncertainties, is $\sigma_{0}=4.6 \%$.

It has been already shown ${ }^{[78,79]}$ that, in the case of axis-symmetric beam distributions as in the LMJ or Orion facilities, the elliptical laser intensity profile allows for better non-uniformities with respect to circular shapes. For the sake of comparison we have also shown in Figure 5 the average (red dashed curve) and the intrinsic non-uniformities (blue dashed curve) calculated using a circular intensity profile. In this case it is assumed that $\Delta_{\mathrm{a}}=\Delta_{\mathrm{b}}=450 \mu \mathrm{m}$ in such a way as to have the same focal spot surface at $I_{0} / e\left(450^{2}=320 \times\right.$ 640). It is found that for the capsule radius $r_{0}=320 \mu \mathrm{m}$ the non-uniformity provided by the circular profile is almost double that of the elliptical case. These results confirm that the elliptical profile provides better results than the circular one for capsule radius $r_{0}<450 \mu \mathrm{m}$.

The specific configurations given by the 10 or 20 quads located in the second ring $\left(49^{\circ}\right)$ of the LMJ facility are not

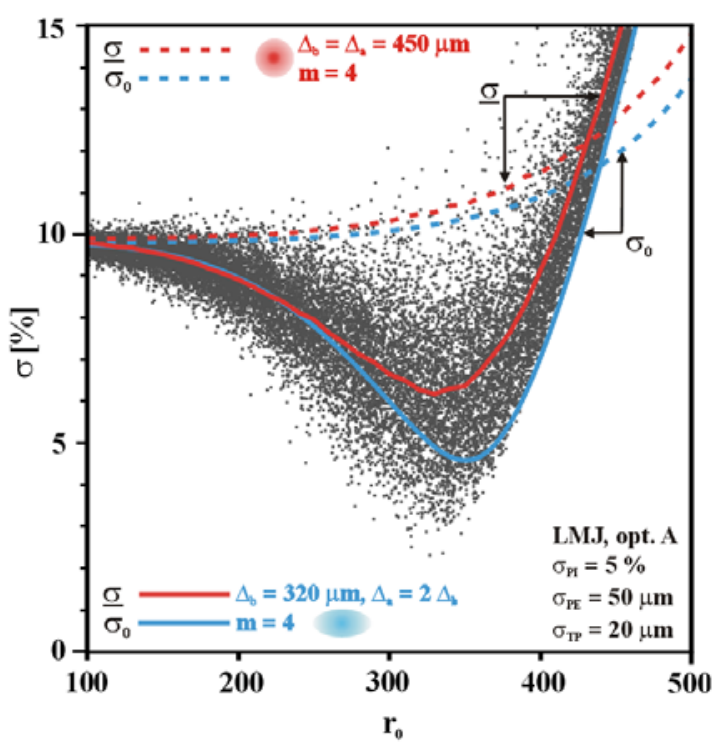

Figure 5. Average illumination non-uniformities $\underline{\sigma}$ (red curves) and intrinsic non-uniformities $\sigma_{0}$ (blue curves) as a function of the capsule radius $r_{0}$ evaluated for the LMJ configuration (option A). Continuous and dashed curves refer to the elliptical and circular laser intensity profile, respectively.

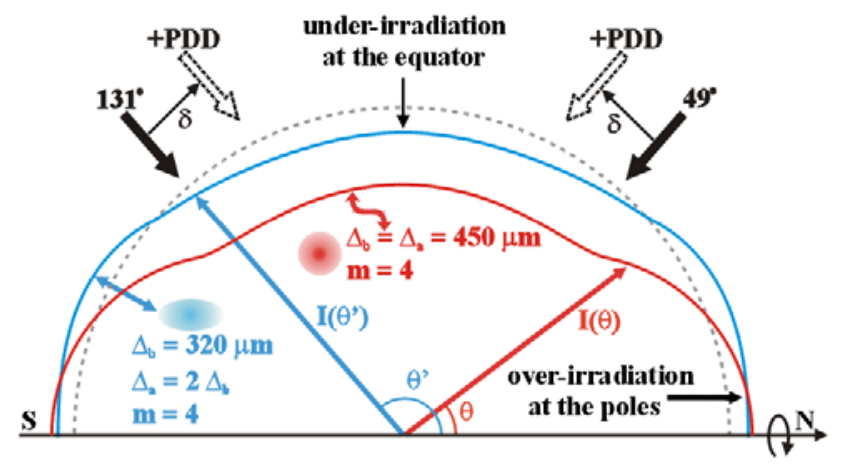

Figure 6. Polar plot of the intensity profile $I(\theta)$ provided by two axissymmetric laser beams illuminating a capsule of radius $r_{0}=320 \mu \mathrm{m}$. The laser intensity profiles are elliptical (red) and circular (blue), while the dashed circle is the reference of a perfectly uniform irradiation.

optimized for direct drive irradiation. Nevertheless, it is worth noting that the polar angle of $49^{\circ}$ is relatively close to the optimum value, $\theta_{\mathrm{S}}=54.7^{\circ}$, as found by Schmitt ${ }^{[70]}$ for optimization of a two-ring configuration assuming that the

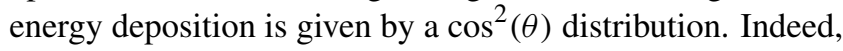
the LMJ configuration provides an over-irradiation of the two polar caps in detriment of the under-irradiation of the equatorial band. This is shown in the polar plot of Figure 6, where the radial distance - which has been set proportionally to the intensity $I(\theta)$ - is shown as a function of capsule latitude $\theta \epsilon[0, \pi]$. The calculations have been performed for an axis-symmetric beam distribution for the elliptical and circular laser intensity profiles and a capsule radius $r_{0}=$ $320 \mu \mathrm{m}$. Both intensity profiles cause an under-irradiation 


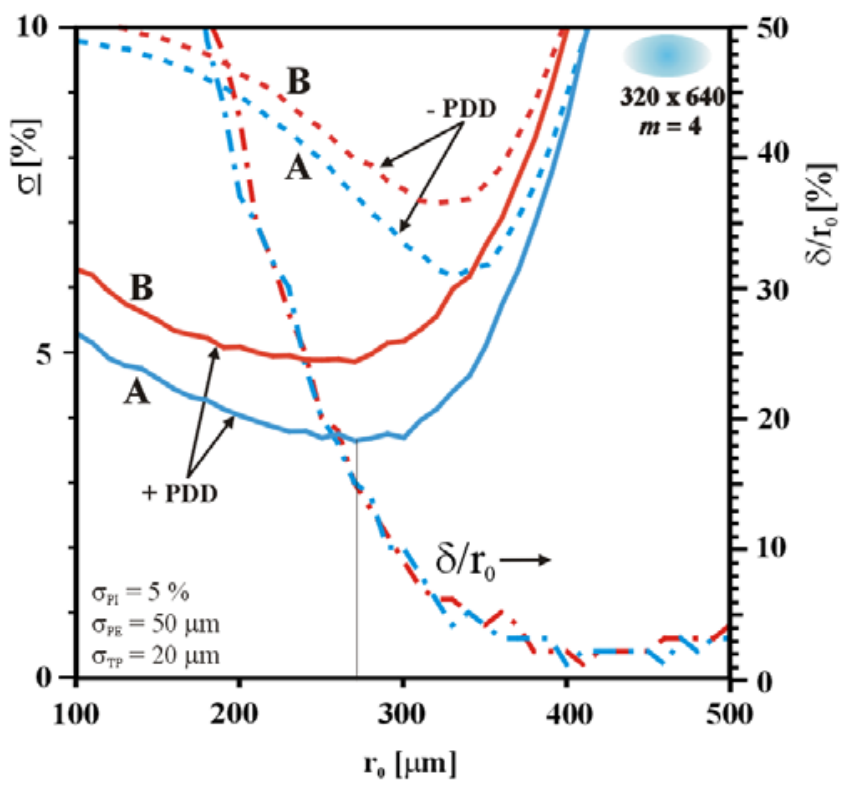

Figure 7. Average irradiation non-uniformity $\bar{\sigma}$ as a function of the capsule radius $r_{0}$ for the LMJ options $\mathrm{A}$ (blue) and $\mathrm{B}$ (red) with (continuous) and without (dashed) applying PDD. In the cases applying PDD, the optimum PDD parameter $\delta / r_{0}$ is also shown.

of the equatorial area but - due to geometrical factors - the elliptical laser spot provides a more uniform radial intensity that better approximates the perfectly spherical symmetry which is represented by the dashed circle.

As previously mentioned, to improve the laser-capsule coupling, the polar direct drive technique has been proposed. In this case, the laser beams are re-directed towards the equator by a quantity $\delta$ in order to balance the irradiation between polar and equatorial areas. The displacement $\delta$ is also indicated in the sketch of Figure 6. A parametric study varying the PDD parameter between 0 and $100 \mu \mathrm{m}$ has been performed looking for the optimal PDD parameter $\delta$ that minimizes the average illumination non-uniformity, $\underline{\sigma}$. These non-uniformities $\underline{\sigma}$ are shown as a function of the capsule radius $r_{0}$ in Figure 7 for the two cases $A$ and B. The dashed curves refer to the calculations without PDD $(\delta=0)$, whilst the continuous curves account for the PDD optimization; in these last cases, the corresponding optimal PDD parameter $\delta / r_{0}$ is also shown. In both configurations, the PDD technique improves the results and reduces the minimum non-uniformities by about $40 \%$. The minimum illumination non-uniformities $\underline{\sigma}_{\mathrm{A}}=3.6 \%$ and $\underline{\sigma}_{\mathrm{B}}=4.9 \%$ are reached at the capsule radius $r_{0}=270 \mu \mathrm{m}$, for which the associated optimum PDD parameter is $\delta / r_{0}=15 \%$.

Another set of calculations has been performed to evaluate the sensitivity of the illumination non-uniformity with respect to a variation of the beam uncertainties $\sigma_{\mathrm{PI}}, \sigma_{\mathrm{PE}}$, and $\sigma_{\mathrm{TP}}$. These calculations use the laser intensity profile envisaged for the LMJ facility $\left(\Delta_{\mathrm{a}}=640 \mu \mathrm{m}, \Delta_{\mathrm{b}}=\right.$ $320 \mu \mathrm{m})$, a capsule radius $r_{0}=270 \mu \mathrm{m}$, and a PDD

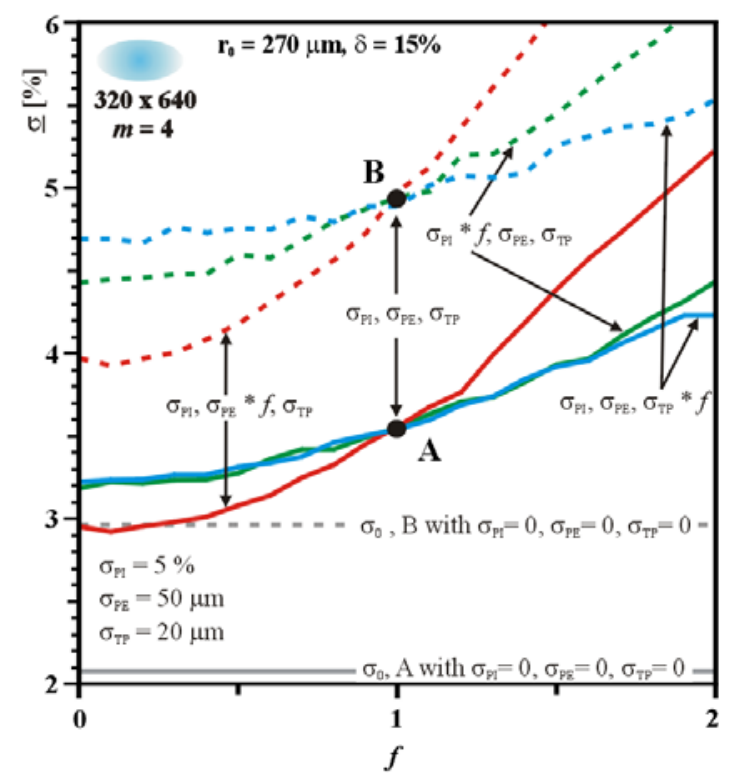

Figure 8. Variation of the average non-uniformity with respect to the laser-capsule uncertainties. Continuous (dashed) curves refer to LMJ option A (B).

parameter $\delta / r_{0}=15 \%$. The average non-uniformities $\underline{\sigma}$ are shown in Figure 8 as a function of an uncertainty scaling parameter $f$, which varies between 0 and 2 . Three series of calculations have been done: (I) keeping constant pointing error $\left(\sigma_{\mathrm{PE}}=50 \mu \mathrm{m}\right)$ and target positioning $\left(\sigma_{\mathrm{TP}}=20 \mu \mathrm{m}\right)$ while varying the power imbalance from zero to double the nominal value $\left(\sigma_{\mathrm{PI}}=5 \%\right)$, i.e., considering $f \sigma_{\mathrm{PI}}, \sigma_{\mathrm{PE}}$, and $\sigma_{\mathrm{TP}}$;(II) varying only the pointing error $\left(\sigma_{\mathrm{PI}}, f \sigma_{\mathrm{PE}}, \sigma_{\mathrm{TP}}\right)$; and (III) with only variation of the target positioning $\left(\sigma_{\mathrm{PI}}, \sigma_{\mathrm{PE}}\right.$, $\left.f \sigma_{\mathrm{TP}}\right)$. The ensemble of the results is shown in Figure 8. As a comparison the two intrinsic values $\sigma_{0}$ (horizontal gray lines) evaluated for the LMJ options A and B have been added. The largest gradient of the average non-uniformity $\underline{\sigma}$ is associated with the variation of the pointing error. This makes these two laser-capsule configurations more sensitive to the pointing error $\left(\sigma_{\mathrm{PE}}\right)$ rather than the other two error sources (power imbalance and target positioning).

A final detailed parametric study has been performed to evaluate the sensitivity of the average illumination nonuniformity to a variation of the PDD parameter $\delta$ and of the super-Gaussian exponent $m$ of the laser intensity profile. As in the previous case, the capsule radius has been set to $r_{0}=$ $270 \mu \mathrm{m}$ and the elliptical intensity profile is characterized by the widths $\Delta_{\mathrm{b}}=320 \mu \mathrm{m}$ and $\Delta_{\mathrm{a}}=2 \Delta_{\mathrm{b}}$. The average nonuniformity, which takes into account the beam uncertainties, is shown as a function of the parameters $\delta$ and $m$ in Figure 9 for LMJ option A (top frame) and B (bottom). The shadowed areas indicate the regions where the non-uniformities are within $10 \%$ closer to their minimum values $\sigma_{\min }(3.6 \%$ for option A and $4.9 \%$ for option B). It is thus shown that both systems tolerate a relatively large variation of the superGaussian exponent $3<m<5$ and a variation of about 

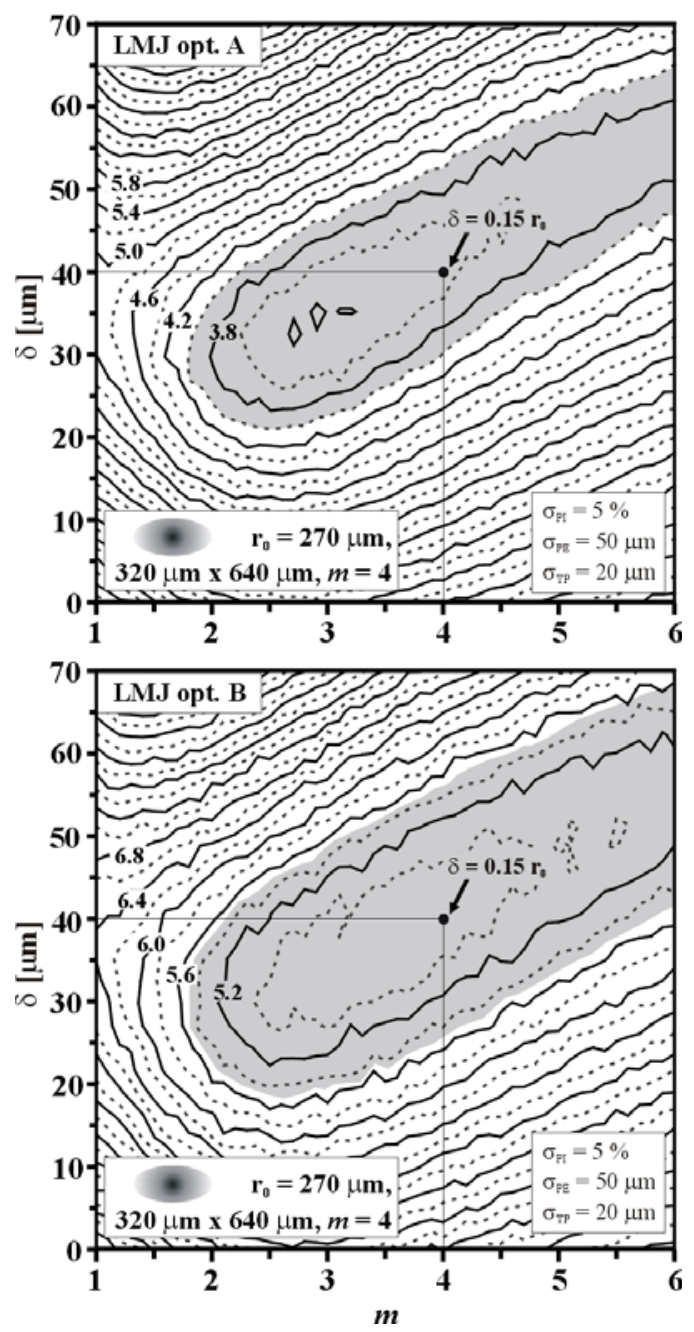

Figure 9. Average non-uniformity as a function of the PDD parameter $\delta$ and of the super-Gaussian exponent $m$ of the laser intensity profile.

$\pm 10 \mu \mathrm{m}$ of the PDD parameter while still providing a nonuniformity smaller than $1.1 \underline{\sigma}_{\min }$.

\section{3D hydrodynamic simulations}

Detailed two-dimensional (2D) hydrodynamic numerical simulations are usually employed to analyse the irradiation, compression, ignition, and thermonuclear burn wave propagation in an ICF capsule. Nevertheless, most actual lasertarget configurations are intrinsically three-dimensional (3D) systems, and these have motivated the development of 3D hydrodynamic numerical tools ${ }^{[80-85]}$. Three-dimensional aspects can play a role also in the present cases considered in this paper that use a limited number of quads. This is especially true in option B, where only five beams are located in each axis-symmetric ring. Recently, using the 3D version of the MULTI ${ }^{[55]}$ code, the uniformity of the irradiation provided by the LMJ facility has been analysed. Here, we

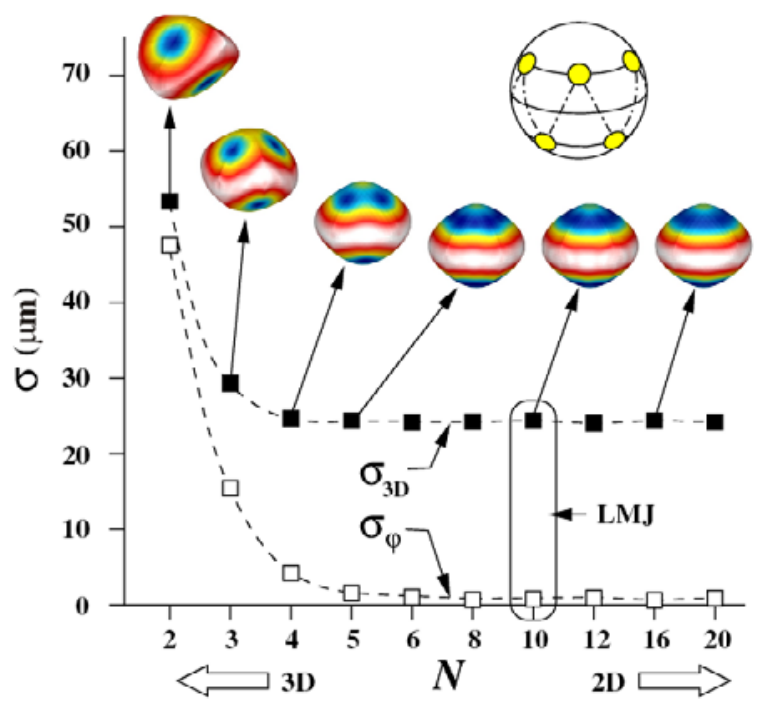

Figure 10. $\sigma_{3 \mathrm{D}}$ (black squares, $\mathbf{\square}$ ) and $\sigma_{\varphi}$ (white squares, $\square$ ) at $t=12 \mathrm{ns,}$ as a function of the number of quads, $N$. Rings of opposite hemispheres are rotated against each other by an angle of $180^{\circ} / \mathrm{N}$.

report only on a few results of a much larger and detailed analysis $^{[86]}$. The 3D version of the MULTI code assumes a non-structured Lagrangian mesh (tetrahedral elements) and accounts for flux-limited $(10 \%)$ thermal heat conduction, tabulated equations of state, and a $3 \mathrm{D}$ ray-tracing package for the laser energy deposition.

A first issue is when a 3D configuration can be correctly described as a 2D axis-symmetric problem. For this purpose, a configuration with a number $N$ of laser beams in each ring has been considered. In these calculations, the spherical capsule described in Section 3 is illuminated by beams from rings at $49^{\circ}$ and $131^{\circ}$, aligned to the target centre $(\delta=0)$, and with a Gaussian radial shape characterized by a full width at half maximum (FWHM) of $1356 \mu \mathrm{m}$. The DT shell is followed in time and a mean radial position is defined as

$R(\theta, \varphi, t)=\int_{D T} \rho(r, \theta, \varphi, t) r^{3} d r / \int_{D T} \rho(r, \theta, \varphi, t) r^{2} d r$.

For a pure axis-symmetric problem, $R$ does not depend on azimuthal angle $\varphi$. Here, it is assumed that departure from perfectly sphericity of the surface defined by the radius $R$ is representative of the non-uniformity produced by the laser energy deposition. This surface has been decomposed in spherical harmonics providing the corresponding timedependent coefficients. Then, these coefficients have been used to measure the azimuthal $\sigma_{\varphi}(t)$ and the polar $\sigma_{\theta}(t)$ components of the total root-mean-square non-uniformity $\sigma_{3 D}^{2}=\sigma_{\varphi}^{2}+\sigma_{\theta}^{2}$ associated to this interface.

The results of these $3 \mathrm{D}$ calculations are summarized in Figures 10 and 11, where we show the values of $\sigma_{3 \mathrm{D}}$ and $\sigma_{\phi}$ evaluated at $t=12 \mathrm{~ns}$, approximately the time when the shell radius reduces to one half of its initial value. Figure 10 corresponds to laser beams arrangements where 


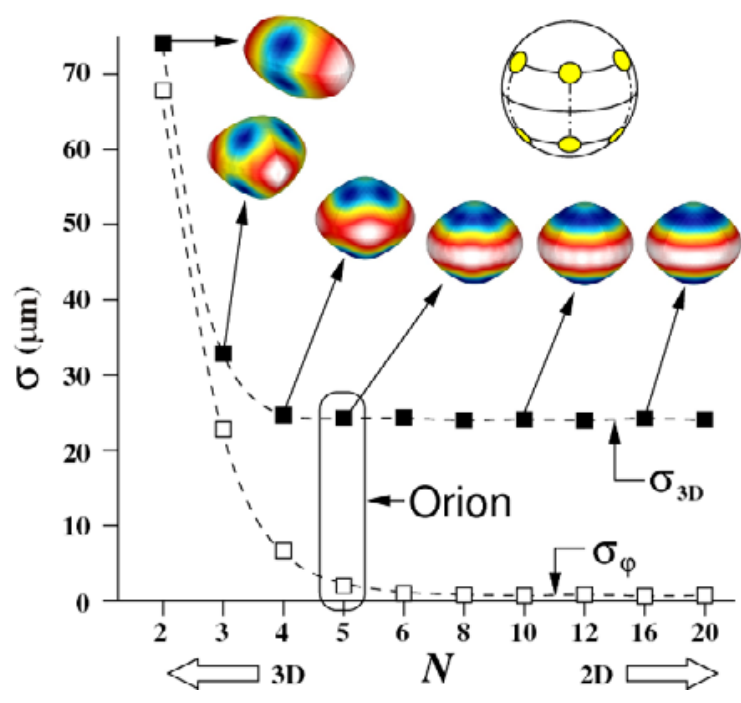

Figure 11. $\sigma_{3 \mathrm{D}}(\boldsymbol{\square})$ and $\sigma_{\varphi}(\square)$ evaluated at $t=12 \mathrm{~ns}$, as a function of the number of quads, $N$. Rings of opposite hemispheres are symmetric with respect to the equatorial plane.

the rings of opposite hemispheres are rotated each other by an angle $18^{\circ} / N$ (e.g., the LMJ facility with $N=10$ ), while Figure 11 corresponds to symmetric arrangements (e.g., the Orion facility, $N=5$ ). The insets in the figures show the shape of the DT-ablator interface evaluated at $t=12 \mathrm{~ns}$. The colours indicate the distortion - inversely related to the driver pressure - in terms of radius (white/blue for large/small values). For extremely small number of beams, there is a clear triaxiality. In Figure 10 one can recognize tetrahedral $(N=2)$ and hexahedral $(N=3)$ shapes. In Figure 11 , for $N=2$, the four quads are in the same meridian plane, and the compressed shape is elongated along the perpendicular direction. In all these cases, polar, azimuthal, and total distortions are of the same order. For large values of $N$, the configuration converges to an axis-symmetric one, the same for both types of laser arrangement. These calculations have been done without PDD correction $(\delta=0)$, and as a consequence a polar overpressure appears. The small residual value $\sigma_{\varphi} \leqslant 1 \mu \mathrm{m}$, for large $N$, is due to the spatial discretization and the numerical noise associated to the Monte Carlo nature of the ray-tracing algorithm. It is noteworthy that the transition between three-dimensionality and two-dimensionality occurs at relatively small laser beam numbers, $N$. For $N=5$, the values of $\sigma_{\phi}$ are $1.55 \mu \mathrm{m}$ and $2.0 \mu \mathrm{m}$, just above the numerical noise. For $N \geqslant 8$, the results are no longer distinguishable. This fast approach to the 2D axial symmetry justifies the use of 2D codes to treat accurately option $\mathrm{A}$ and, in an approximate but reasonable way, option $\mathrm{B}$. These conclusions hold when spots of adjacent beams have enough overlapping, provided that uncertainties in beam power balance and pointing accuracy can be neglected. It must be mentioned that, for configurations where the beam size has been reduced
(FWHM $\approx 1000 \mu \mathrm{m}), 3 \mathrm{D}$ effects occur, and azimuthal distortions can becomes significant even for $N \approx 5^{[86]}$.

\section{Conclusions}

The Laser MegaJoule facility has been considered in the context of the shock ignition scheme. Two laser beam configurations have been analysed. A first option (A) uses 20 quads - 80 laser beams $(600 \mathrm{~kJ}, 200 \mathrm{TW})$ locate at the second ring of the LMJ facility - for the compression of the capsule, making available the remaining 24 quads - 96 laser beams $(720 \mathrm{~kJ}, 240 \mathrm{TW})$ - for the additional shock ignition pulse. A second option (B) envisages the possibility to use only 10 quads for the compression phase and 34 quads for the compression and SI phases. The total available laser power is $440 \mathrm{TW}$ at $3 \omega(\lambda=351 \mathrm{~nm})$.

A classical ICF capsule - devoted to the central ignition scheme - has been used in the context of the shock ignition scheme. A set of mono-dimensional numerical simulations has been performed to enlighten some aspect of the shock ignition scheme. For this specific capsule it is found that the threshold power in the shock ignition pulse is about 250 TW. Nevertheless, assuming that all this power is incident to the surface of the critical density provides incident intensity larger than $10^{16} \mathrm{TW} \mathrm{cm}^{-2}$. At these large intensities $\left(I \lambda^{2}>\right.$ $10^{15} \mathrm{~W} \mathrm{~cm}^{-2} \mu \mathrm{m}^{2}$ ) we expect saturation of dangerous laserplasma instabilities (SRS, SBS, and TPD) that modify the laser energy deposition mechanism. In this new regime, a large fraction of the laser energy is transferred to highenergetic electrons, and the photon penetration depth is limited to a quarter of the critical density $\left(\rho_{\mathrm{c}} / 4\right)$, instead of the classical limit, $\rho_{\mathrm{c}}$. These physical mechanisms are not included in our numerical tools; however, we performed some calculations to estimate the effect caused by limiting the deposition of the laser energy in the region at lower density $\left(\rho<\rho_{\mathrm{c}} / 4\right)$. To mimic this effect, the light wavelength during the shock ignition pulse has been artificially doubled $\left(\lambda_{\mathrm{SI}}=2 \lambda\right)$; thus, because $\rho_{\mathrm{c}} \alpha \lambda^{-2}$, the critical density becomes a quarter. As expected, this affects negatively the power threshold in the shock ignition pulse that now increases to about $400 \mathrm{TW}$. This should be considered as a pessimistic estimation. In fact, none of the positive effects associated with the high-energetic electrons are included in our calculations.

The second issue addressed in the paper concerns the irradiation uniformity provided during the first few ns of the foot pulse. First, it has been shown that the elliptical laser intensity profile of the LMJ facility provides better results in comparison to the usually circular profile. The two LMJ options $\mathrm{A}$ and $\mathrm{B}$ have been considered, taking into account beam uncertainties such as quad-to-quad power imbalance $\left(\sigma_{\mathrm{PI}}=5 \%\right)$, pointing error $\left(\sigma_{\mathrm{PE}}=50 \mu \mathrm{m}\right)$, and target positioning $\left(\sigma_{\mathrm{TP}}=20 \mu \mathrm{m}\right)$. Both of these configurations cause an over-irradiation of the capsule polar regions in 
detriment to the equatorial area. In order to improve these schemes, the polar direct drive technique has been applied to optimize the irradiation uniformity. It has been found that for the elliptical laser intensity profile $\left(\Delta_{\mathrm{b}}=320 \mu \mathrm{m}\right.$, $\Delta_{\mathrm{a}}=2 \Delta_{\mathrm{b}}, m=4$ ) expected at the LMJ facility the optimal capsule radius is $r_{0}=270 \mu \mathrm{m}$, and this provides an average illumination non-uniformity of $\underline{\sigma}=3.6 \%$ and $4.9 \%$ in case A and case $\mathrm{B}$, respectively. These minimum non-uniformities correspond to the use of a PDD parameter $\delta / r_{0}=15 \%$. This capsule radius is relatively small in comparison to the available LMJ energy and the requirements for typical ignition capsule designs; however, bigger capsules could be envisaged assuming larger focal spots provided by either defocusing of the laser beams or using an alternative set of phase plates.

A 3D version of the code MULTI has been used to perform a set of preliminary hydrodynamic calculations. The LMJ options A and B have been considered in these calculations, and the laser irradiation uniformity has been split into the azimuthal and polar components by means of decomposition in spherical harmonics. For the analysed laser-capsule configuration - with the laser intensity profile that reduces to $1 / e$ at the initial capsule radius - it is found that the azimuthal component is negligible in the case of option A (ten beams per hemisphere). This encouraging result seems indicates that a 2D analysis is appropriate in option A, while in the second case, option B, it may not be. Of course these conclusions depend on the beam and capsule sizes, and further investigations are needed for specific configurations.

Finally, the two LMJ options A and B involve the use of 10 or 20 quads located in the second rings characterized by the polar angles $49^{\circ}$ and $131^{\circ}$. These options are in many aspects similar to the configuration already available at the Orion facility, where ten laser beams are located at $50^{\circ}$ and $130^{\circ}$. In addition, these ten ns-long laser beams operate at the wavelength $\lambda=0.351 \mu \mathrm{m}(3 \omega)$ as in the LMJ facility. The similarity between the two installations motivates us to stress the opportunity to perform Orion's experiments addressed to PDD issues of interest also for future direct drive LMJ campaigns. Indeed, although of relatively small energy $-5 \mathrm{~kJ}$ in few ns for the ten longpulse Orion beams - this installation is fully adequate for direct drive experiments that may explore the laser-capsule coupling as well as the uniformity and timing of the first shock wave generated during the low-power $(\approx \mathrm{TW})$ ns-long foot pulse needed to control the initial imprint phase of an ICF implosion, thus helping to underwrite modelling of polar direct drive implosions.

\section{Acknowledgements}

M. T. and B. C. express their thanks to Daniel Bouche for the support given to this work. R. R. was partially supported by the EURATOM/CIEMAT association in the framework of the 'IFE Keep-in-Touch Activities'. S. W. acknowledges support from the Czech Science Foundation (Project No. CZ.1.07/2.3.00/20.0279) and from ELI (Project No. CZ.1.05/1.1.00/02.0061).

\section{References}

1. J. Lindl, Phys. Plasmas 2, 3933 (1995).

2. J. D. Lindl, P. Amendt, R. L. Berger, S. G. Glendinning, S. H. Glenzer, S. W. Haan, R. L. Kauffman, O. L. Landen, and L. J. Suter, Phys. Plasmas 11, 339 (2004).

3. S. Atzeni and J. Meyer-ter-Vehn, The Physics of Inertial Fusion (Oxford University Press, Oxford, 2004).

4. S. E. Bodner, D. G. Colombant, J. H. Gardner, R. H. Lehmberg, S. P. Obenschain, L. Phillips, A. J. Schmitt, J. D. Sethian, R. L. McCrory, W. Seka, C. P. Verdon, J. P. Knauer, B. B. Afeyan, and H. T. Powell, Phys. Plasmas 5, 1901 (1998).

5. J. H. Nuckolls, L. Wood, A. Thiessen, and G. B. Zimmermann, Nature 239, 129 (1972).

6. R. D. Richtmyer, Commun. Pure Appl. Math. 13, 297 (1960).

7. E. E. Meshkov, Fluid Dyn. 4, 101 (1969).

8. L. Rayleigh, Scientific Papers. Vol. II (Dover, New York, 1965).

9. G. I. Taylor, Proc. R. Soc. London, Ser. A 201, 192 (1950).

10. R. Betti and C. Zhou, Phys. Plasmas 12, 110702 (2005).

11. N. G. Basov, S. Yu. Gus'kov, and L. P. Feoktistov, J. Sov. Laser Res. 13, 396 (1992).

12. M. Tabak, J. Hammer, M. E. Glinsky, W. L. Kruer, S. C. Wilks, J. Woodworth, E. M. Campbell, M. D. Perry, and R. J. Mason, Phys. Plasmas 1, 1626 (1994).

13. M. Roth, T. E. Cowan, M. H. Key, S. P. Hatchett, C. Brown, W. Fountain, J. Johnson, D. M. Pennington, R. A. Snavely, S. C. Wilks, K. Yasuike, H. Ruhl, F. Pegoraro, S. V. Bulanov, E. M. Campbell, M. D. Perry, and H. Powell, Phys. Rev. Lett. 86, 436 (2001).

14. S. Yu. Gus'kov, Quantum Electronics 31, 885 (2001).

15. M. Temporal, J. J. Honrubia, and S. Atzeni, Phys. Plasmas 9, 3098 (2002).

16. B. M. Hegelich, B. J. Albright, J. Cobble, K. Flippo, S. Letzring, M. Paffett, H. Ruhl, J. Schreiber, R. K. Schulze, and J. C. Fernandez, Nature 439, 441 (2006).

17. J. C. Fernandez, J. J. Honrubia, B. J. Albright, K. A. Flippo, D. C. Gautier, B. M. Hegelich, M. J. Schmitt, M. Temporal, and L. Yin, Nucl. Fusion 49, 065004 (2009).

18. V. A. Shcherbakov, Sov. J. Plasma Phys. 9, 240 (1983).

19. R. Betti, C. D. Zhou, K. S. Anderson, L. J. Perkins, W. Theobald, and A. A. Solodov, Phys. Rev. Lett. 98, 155001 (2007).

20. A. J. Schmitt, J. W. Bates, S. P. Obenschain, S. T. Zalesak, and D. E. Fyfe, Phys. Plasmas 17, 042701 (2010).

21. M. Lafon, X. Ribeyre, and G. Schurtz, Phys. Plasmas 17, 052704 (2010).

22. L. Hallo, M. Olazabal-Loume, X. Ribeyre, V. Drean, G. Schurtz, J. L. Feugeas, J. Breil, Ph. Nicolai, and P. H. Maire, Plasma Phys. Control. Fusion 51, 014001 (2009).

23. X. Ribeyre, G. Schurtz, M. Lafon, S. Galera, and S. Weber, Plasma Phys. Control. Fusion 51, 015013 (2009).

24. R. Lehmberg and J. Goldhar, Fusion Tech. 11, 532 (1987).

25. B. Canaud and F. Garaude, Nucl. Fusion 45, L43 (2005).

26. H. A. Rose, D. F. DuBois, and B. Bezzerides, Phys. Rev. Lett. 58, 2547 (1987).

27. K. Estabrook, J. Harte, E. Campbell, F. Ze, D. Phillion, M. Rosen, and J. Larsen, Phys. Rev. Lett. 46, 724 (1981).

28. R. Yan, A. Maximov, C. Ren, and F. Tsung, Phys. Rev. Lett. 103, 175002 (2009).

29. O. Klimo, V. T. Tikhonchuk, X. Ribeyre, G. Schurtz, C. Riconda, S. Weber, and J. Limpouch, Phys. Plasmas 18, 082709 (2011). 
30. R. Yan, A. Maximov, and C. Ren, Phys. Plasmas 17, 052701 (2010).

31. H. Vu, D. DuBois, D. Russell, and J. Myatt, Phys. Plasmas 17, 072701 (2010).

32. O. Klimo, S. Weber, V. T. Tikhonchuk, and J. Limpouch, Plasma Phys. Control. Fusion 52, 055013 (2010).

33. W. Theobald, R. Betti, C. Stoeckl, K. S. Anderson, J. A. Delettrez, V. Yu. Glebov, V. N. Goncharov, F. J. Marshall, D. N. Maywar, R. L. McCrory, D. D. Meyerhofer, P. B. Radha, T. C. Sangster, W. Seka, D. Shvarts, V. A. Smalyuk, A. A. Solodov, B. Yaakobi, C. D. Zhou, J. A. Frenje, C. K. Li, F. H. Séguin, R. D. Petrasso, and L. J. Perkins, Phys. Plasmas 15, 056306 (2008).

34. S. D. Baton, M. Koenig, E. Brambrink, H. P. Schlenvoigt, C. Rousseaux, G. Debras, S. Laffite, P. Loiseau, F. Philippe, X. Ribeyre, and G. Schurtz, Phys. Rev. Lett. 108, 195002 (2012).

35. W. Theobald, R. Nora, M. Lafon, A. Casner, X. Ribeyre, K. S. Anderson, R. Betti, J. A. Delettrez, J. A. Frenje, V. Yu. Glebov, O. V. Gotchev, M. Hohenberger, S. X. Hu, F. J. Marshall, D. D. Meyerhofer, T. C. Sangster, G. Schurtz, W. Seka, V. A. Smalyuk, C. Stoeck, and B. Yaakobi, Phys. Plasmas 19, 102706 (2012).

36. D. Batani, L. A. Gizzi, P. Koester, L. Labate, J. Honrubia, L. Antonelli, A. Morace, L. Volpe, J. J. Santos, G. Schurtz, S. Hulin, X. Ribeyre, P. Nicolai, B. Vauzour, F. Dorchies, W. Nazarov, J. Pasley, M. Richetta, K. Lancaster, C. Spindloe, M. Tolley, D. Neely, M. Kozlov'a, J. Nejdl, B. Rus, J. Wol owski, and J. Badziak, Nukleonika 57, 3 (2012).

37. P. Koester, L. Antonelli, S. Atzeni, J. Badziak, F. Baffigi, D. Batani, C. A. Cecchetti, T. Chodukowski, F. Consoli, G. Cristoforetti, R. De Angelis, G. Folpini, L. A. Gizzi, Z. Kalinowska, E. Krousky, M. Kucharik, L. Labate, T. Levato, R. Liska, G. Malka, Y. Maheut, A. Marocchino, P. Nicolai, T. O’Dell, P. Parys, T. Pisarczyk, P. Raczka, O. Renner, Y. J. Rhee, X. Ribeyre, M. Richetta, M. Rosinski, L. Ryc, J. Skala, A. Schiavi, G. Schurtz, M. Smid, C. Spindloe, J. Ullschmied, J. Wolowski, and A. Zaras, Plasma Phys. Control. Fusion 55, 124045 (2013).

38. M. Hohenberger, W. Theobald, S. X. Hu, K. S. Anderson, R. Betti, T. R. Boehly, A. Casner, D. E. Fratanduono, M. Lafon, D. D. Meyerhofer, R. Nora, X. Ribeyre, T. C. Sangster, G. Schurtz, W. Seka, C. Stoeckl, and B. Yaakobi, Phys. Plasmas 21, 022702 (2014).

39. C. Goyon, S. Depierreux, V. Yahia, G. Loisel, C. Baccou, C. Courvoisier, N. G. Borisenko, A. Orekhov, O. Rosmej, and C. Labaune, Phys. Rev. Lett. 111, 235006 (2013).

40. G. H. Miller, E. I. Moses, and C. R. Wuest, Nucl. Fusion 44, 228 (2004).

41. E. I. Moses, R. N. Boyd, B. A. Remington, C. J. Keane, and R. Al-Ayat, Phys. Plasmas 16, 041006 (2009).

42. C. Cavailler, N. Fleurot, T. Lonjaret, and J. M. Di-Nicola, Plasma Phys. Control. Fusion 46, B135 (2004).

43. C. Lion, Journal of Physics: Conference Series 244, 012003 (2010).

44. N. Hopps, C. Danson, S. Duffield, D. Egan, S. Elsmere, M. Girling, E. Harvey, D. Hillier, M. Norman, S. Parker, P. Treadwell, D. Winter, and T. Bett, Appl. Opt. 52, 3597 (2013).

45. S. Jacquemot, F. Amiranoff, S. D. Baton, J. C. Chanteloup, C. Labaune, M. Koenig, D. T. Michel, F. Perez, H. P. Schlenvoigt, B. Canaud, C. Cherfils Clerouin, G. Debras, S. Depierreux, J. Ebrardt, D. Juraszek, S. Lafitte, P. Loiseau, J. L. Miquel, F. Philippe, C. Rousseaux, N. Blanchot, C. B. Edwards, P. Norreys, S. Atzeni, A. Schiavi, J. Breil, J. L. Feugeas, L. Hallo, M. Lafon, X. Ribeyre, J. J. Santos, G. Schurtz, V. Tikhonchuk, A. Debayle, J. J. Honrubia, M. Temporal, D. Batani, J. R. Davies, F. Fiuza, R. A. Fonseca, L. O. Silva, L. A. Gizzi, P. Koester, L. Labate, J. Badziak, and O. Klimo, Nucl. Fusion 51, 094025 (2011).
46. S. Skupsky, J. A. Marozas, R. S. Craxton, R. Betti, T. J. B. Collins, J. A. Delettrez, V. N. Goncharov, P. W. McKenty, P. B. Radha, J. P. Knauer, F. J. Marshall, D. R. Harding, J. D. Kilkenny, D. D. Meyerhofer, T. C. Sangster, and R. L. McCrory, Plasma Phys. 11, 2763 (2004).

47. R. S. Craxton, F. J. Marshall, M. J. Bonino, R. Epstein, P. W. McKenty, S. Skupsky, J. A. Delettrez, I. V. Igumenshchev, D. W. Jacobs-Perkins, J. P. Knauer, J. A. Marozas, P. B. Radha, and W. Seka, Phys. Plasmas 12, 056304 (2005).

48. B. Canaud, X. Fortin, F. Garaude, C. Meyer, and F. Philippe, Laser Part. Beam 22, 109 (2004).

49. B. Canaud, F. Garaude, P. Ballereau, J. L. Bourgade, C. Clique, D. Dureau, M. Houry, S. Jaouen, H. Jourdren, N. Lecler, L. Masse, A. Masson, R. Quach, R. Piron, D. Riz, J. Van der Vliet, M. Temporal, J. A. Delettrez, and P. W. McKenty, Plasma Phys. Control. Fusion 49, B601 (2007).

50. P. B. Radha, J. A. Marozas, F. J. Marshall, A. Shvydky, T. J. B. Collins, V. N. Goncharov, R. L. McCrory, P. W. McKenty, D. D. Meyerhofer, T. C. Sangster, and S. Skupsky, Phys. Plasmas 19, 082704 (2012).

51. P. B. Radha, F. J. Marshall, J. A. Marozas, A. Shvydky, I. Gabalski, T. R. Boehly, T. J. B. Collins, R. S. Craxton, D. H. Edgell, R. Epstein, J. A. Frenje, D. H. Froula, V. N. Goncharov, M. Hohenberger, R. L. McCrory, P. W. McKenty, D. D. Meyerhofer, R. D. Petrasso, T. C. Sangster, and S. Skupsky, Phys. Plasmas 20, 056306 (2013).

52. V. Brandon, B. Canaud, M. Primout, S. Laffite, and M. Temporal, Laser Part. Beam 31, 141 (2013).

53. V. Brandon, B. Canaud, M. Temporal, and R. Ramis, Low Initial Aspect-Ratio Direct-Drive target designs for shock- or self-ignition in the context of the laser Megajoule, submitted to Nucl Fusion (2014).

54. M. Temporal, V. Brandon, B. Canaud, J. P. Didelez, R. Fedosejevs, and R. Ramis, Nucl. Fusion 52, 103011 (2012).

55. R. Ramis, R. Schmaltz, and J. Meyer-ter-Vehn, Comput. Phys. Commun. 49, 475 (1988).

56. R. Ramis, K. Eidmann, J. Meyer-ter-Vehn, and S. Hüller, Comput. Phys. Commun. 183, 637 (2012).

57. W. L. Kruer, The Physics of Laser-Plasma Interactions (Addison-Wesley, Reading, MA, 1988).

58. C. Riconda, S. Weber, V. T. Tikhonchuk, and A. Heron, Phys. Plasmas 18, 092701 (2011).

59. S. Weber, C. Riconda, O. Klimo, A. Heron, and V. T. Tikhonchuk, Phys. Rev. E. 85, 016403 (2012).

60. O. Klimo and V. T. Tikhonchuk, Plasma Phys. Control. Fusion 55, 095002 (2013).

61. W. Theobald, R. Nora, M. Lafon, A. Casner, X. Ribeyre, K. S. Anderson, R. Betti, J. A. Delettrez, J. A. Frenje, V. Yu. Glebov, O. V. Gotchev, M. Hohenberger, S. X. Hu, F. J. Marshall, D. D. Meyerhofer, T. C. Sangster, G. Schurtz, W. Seka, V. A. Smalyuk, C. Stoeckl, and B. Yaakobi, Phys. Plasmas 19, 102706 (2012).

62. R. E. Kidder, Nucl. Fusion 16, 405 (1976).

63. A. Bell and M. Tzoufras, Plasma Phys. Control. Fusion 53, 045010 (2011).

64. S. Gus'kov, X. Ribeyre, M. Touati, J. L. Feugeas, Ph. Nicolai, and V. Tikhonchuk, Phys. Rev Lett. 109, 255004 (2012).

65. X. Ribeyre, S. Gus'kov, J. L. Feugeas, Ph. Nicolai, and V. T. Tikhonchuk, Phys. Plasmas 20, 062705 (2013).

66. B. Canaud, S. Laffite, V. Brandon, and M. Temporal, Laser Part. Beam 30, 183 (2012).

67. S. Atzeni, A. Schiavi, and A. Marocchino, Plasma Phys. Control. Fusion 53, 035010 (2011).

68. M. Temporal and B. Canaud, Eur. Phys. J. D. 55, 139 (2009). 
69. S. Skupsky and K. Lee, J. Appl. Phys. 54, 3662 (1983).

70. A. J. Schmitt, Appl. Phys. Lett. 44, 399 (1984).

71. M. Temporal, B. Canaud, W. J. Garbett, and R. Ramis, Comparison between illumination model and hydrodynamic simulation for a Direct Drive laser irradiated capsule (2014), in preparation.

72. M. Murakami, Appl. Phys. Lett. 66, 1587 (1995).

73. M. Murakami, K. Nishihara, and H. Azechi, J. Appl. Phys. 74, 802 (1993).

74. B. Canaud, X. Fortin, N. Dague, and J. Bocher, Phys. Plasmas 9, 4252 (2002).

75. M. Temporal, B. Canaud, and B. J. Le Garrec, Phys. Plasmas 17, 022701 (2010).

76. M. Temporal, B. Canaud, S. Laffite, B. J. Le Garrec, and M. Murakami, Phys. Plasmas 17, 064504 (2010).

77. M. Temporal, R. Ramis, B. Canaud, V. Brandon, S. Laffite, and B. J. Le Garrec, Plasma Phys. Control. Fusion 53, 124008 (2011).

78. M. Temporal, B. Canaud, W. J. Garbett, and R. Ramis, Phys. Plasmas 21, 012710 (2014).

79. M. Temporal, B. Canaud, W. J. Garbett, F. Philippe, and R. Ramis, Eur. Phys. J. D. 67, 205 (2013).

80. R. Ramis, Phys. Plasmas 20, 082705 (2013).
81. M. M. Marinak, G. D. Kerbel, J. M. Koning, M. V. Patel, S. M. Sepke, M. S. McKinley, M. J. O’Brien, R. J. Procassini, and D. Munro, EPJ Web of Conferences 59, 06001 (2013).

82. M. Gittings, R. Weaver, M. Clover, T. Betlach, N. Byrne, R. Coker, E. Dendy, R. Hueckstaedt, K. New, W. R. Oakes, D. Ranta, and R. Stefan, Comput. Sci. Disc. 1, 015005 (2008).

83. B. van der Holst, G. Toth, I. V. Sokolov, K. G. Powell, J. P. Holloway, E. S. Myra, Q. Stout, M. L. Adams, J. E. Morel, S. Karni, B. Fryxell, and R. P. Drake, Astrophys. J. Suppl. 194, 23 (2011)

84. B. Fryxell, K. Olson, P. Ricker, F. X. Timmes, M. Zingale, D. Q. Lamb, P. Macneice, R. Rosner, J. W. Truran, and H. Tufo, Astrophys. J. Suppl. 131, 273 (2000).

85. Jinghong Lia, Chuanlei Zhai, Shuanggui Li, Xin Li, Wudi Zheng, Heng Yong, Qinghong Zeng, Xudeng Hang, Jin Qi, Rong Yang, Juan Cheng, Peng Song, Peijun Gu, Aiqing Zhang, Hengbin An, Xiaowen Xu, Hong Guo, Xiaolin Cao, Zeyao Mo, Wenbing Pei, Song Jiang, and Shao-ping Zhu, EPJ Web of Conferences 59, 06002 (2013).

86. R. Ramis, M. Temporal, B. Canaud, V. Brandon, and S. Laffite, Three-dimensional symmetry analysis of a Direct Drive irradiation scheme for the Laser MegaJoule facility (2014), in preparation. 